\section{HIPERTENSIÓN ARTERIAL EN MENORES DE EDAD DEL HOS- PITAL GENERAL DE ZACATECAS, MÉXICO.} ARTERIAL HYPERTENSION IN MINORS OF THE GENERAL HOSPITAL OF ZACATECAS, MEXICO

\author{
Ulloa-Galván Víctor Manuel'1, De la Torre-Cabral Carlos Alberto,Cortes-Ramírez Juan Ma- \\ nuel $^{2}$, Salazar-de Santiago Alfredo ${ }^{3}$.
}

\section{RESUMEN}

Objetivo: Obtener la prevalencia de hipertensión arterial sistémica en niños en el Hospital General de Zacatecas (HGZ). Identificar género, edad, etiología, diagnóstico, métodos confirmatorios y terapéutica. Además, comparar resultados con la literatura médica.

Métodos: Estudio retrospectivo descriptivo de prevalencias que analizó 5941 expedientes digitales de servicios de cardiología y nefrología pediátrica del HGZ, de agosto 2013 a agosto 2015, en el sistema de cómputo hospitalario, denominado SIGHO. Se seleccionó pacientes con diagnóstico de Hipertensión Arterial Sistémica (HAS), revisándoles género, edad, etiología, método de confirmación diagnóstica, y tratamiento indicado, mediante el computador central y administrativo del hospital, para identificar las consultas y pacientes con los filtros específicos. El análisis de datos se estableció por medio del Software SPSS.19® para Windows.

Resultados: De 5 94I consultas totales, 3277 de Cardiopediatría y 2664 de nefropediatría. Con solo 40 pacientes con HAS, 0,67\%; 25 de ellos masculinos, 62,5\%; una frecuencia asentada entre edades de I-4 años, con $45 \%$. En su etiología la mayoría presentaron coartación de aorta con $57,5 \%$, insuficiencia renal crónica en un $27,5 \%$, solo I niño con tumor renal, I con HAS renovascular y I con HAS primaria, equivalente 2,5\%, y se clasifico 3 pacientes con HAS secundaria sin especificar, $7,5 \%$.

Conclusiones: Resalta la baja prevalencia de HAS pediátrica, de 0,67\%, en casi 6000 consultas, comparativamente con la literatura universal de I,5-3\%; y a casuística con coartación aortica en 57,5\% e insuficiencia renal crónica en $27,5 \%$, y otras patologías renales en menor frecuencia, invirtiendo causas primero la coartación de aorta y después problemas parenquimatosos renales.

\section{ABSTRACT}

Aim: To obtain the prevalence of hypertension in children in the General Hospital of Zacatecas (HGZ). Identify gender, age, etiology, diagnosis, and therapeutic methods Confirmatory. In addition, to compare results with the medical literature.

Methods: Retrospective study that analyzed 5 94I digital records of pediatric cardiology and pediatric nephrology of HGZ, from August 2013 to August 20I5, in the hospital computer system, called SIGHO. Patients were selected with diagnosis of systemic hypertension (HAS), reviewing gender, age, etiology, methods of diagnostic confirmation and treatment indicated by the central and administrative computer of the hospital for identify queries and patients with specific filters. Data analysis was established by the SPSS.19® Software for Windows.

Results: Of 5 94I total views, 3277 and 2664 of pediatric cardiology and pediatric nephrology. With only 40 patients with HAS, 0,67\%; 25 of them male, 62,5\%; main frequency seated between the ages of I-4 years, with $45 \%$. In its etiology most they had aortic coarctation with $57,5 \%$, chronic renal failure in $27,5 \%$, only I child with renal tumor, I with HAS by the renal vasculature and I with HAS primary, equivalent to $2,5 \%$ and 3 patients was classified with HAS secondary unspecified $7,5 \%$.

Conclusions: Highlight the low prevalence of pediatric hypertension, $0,67 \%$, almost 6,000 consultations, compared with world literature 1,5-3\%; Casuistry with coarctation in $57,5 \%$ and chronic renal failure in $27,5 \%$, and other renal diseases less frequently, investing causes aortic coarctation first and then parenchymal kidney problems.

\section{INTRODUCCIÓN}

$\mathrm{L}$ a presión arterial normal se define como la presión sistólica y/o diastólica menor de 90 percentiles para género, edad y estatura; un punto importante de mencionar en este apartado es cuando los rangos se encuentran entre el percentil 90 y 95 , que se considera en que el paciente tendrá mayor riesgo para desarrollar hipertensión. ${ }^{1}$ presión arterial "normal alta" cuya importancia reside
Estudiante de medicina de la Unidad Académica de Medicina Humana, Universidad Autónoma de Zacatecas, Zacatecas, México.

${ }^{2}$ Médico internista. Miembro adscrito al Hospital General de Zacatecas "Luz María Cosío", Zacatecas, México. Docente en la Unidad Académica de Medicina Humana, Universidad Autónoma de Zacatecas.

${ }^{2}$ Medico Cirujano Dentista Maestría en Ciencias de la Salud con Especialidad en Salud Pública. Docente en la Unidad Académica de Medicina Humana, Universidad Autónoma de Zacatecas

Correspondencia a: Víctor Manuel Ulloa Galván Correo electrónico: vicullga@ gmail.com

Palabras clave: Hipertensión, Niños, Epidemiología.

Keywords: Hypertension, child, Epidemiology.

Procedencia y arbitraje: no comisionado, sometido a arbitraje externo.

Recibido para publicación 15 de agosto del 2016 Aceptado para publicación: 04 de Noviembre del 2016

Citar como: Rev Cient Cienc Med 2016;19(2): 27-32 
Se define hipertensión arterial sistémica (HAS) de acuerdo con las recomendaciones actuales del grupo de trabajo de hipertensión de la Academia Americana de Pediatría , como los niveles de tensión arterial sistólica (TAS) o diastólica (TAD) iguales o superiores al percentil 95 (P95) para una determinada edad, sexo y percentil de talla. ${ }^{1}$

La etiología de la hipertensión arterial durante la infancia suele ser de tipo secundaria y en ella $80 \%$ de origen renal. Hasta hace poco tiempo, esta condición se consideraba rara en los infantes, pero cada vez se vuelve más usual encontrar pacientes pediátricos con esta condición, esto por su asociación con la obesidad infantil y los antecedentes familiares de hipertensión arterial sistémica. Lo cual vuelve el diagnóstico algo de suma importancia para poder evitar la cronicidad de este padecimiento, y prevenir las posibles complicaciones tempranas. ${ }^{1}$

La hipertensión tiene una prevalencia del $20 \%$ en el adulto, aunque en la población infantil (de 4 a 15 años de edad), es de 1,5 a 2\%.2 En un gran número de casos la presencia de HAS en la infancia es consecuencia de una serie de patologías subyacentes, (el 80 a $90 \%$ de los niños tienen como causa una enfermedad renal, un $10 \%$ renovascular y un $5 \%$ coartación aórtica), cuya primera manifestación puede ser la elevación de la presión arterial (PA).

Para la clasificación de la hipertensión arterial en niños a partir del año de edad se considera:

- Presión arterial normal: Presión arterial sistólica y diastólica por debajo del percentil 90 para edad, género y estatura.

- Pre-hipertensión (presión arterial normal alta): Presión arterial sistólica o diastólica mayor o igual al percentil 90, y menor al percentil 95.

- Hipertensión arterial: Presión arterial sistólica y/o diastólica mayor o igual al percentil 95.

- Hipertensión arterial Estadío I: Presión arterial sistólica y/o diastólica del percentil 95 al percentil 99, o más de $5 \mathrm{mmHg}$

- Hipertensión arterial Estadío 2: Presión arterial sistólica y/o diastólica mayor del percentil 99, más $5 \mathrm{mmHg}$.

Para la medición de la presión arterial,2,3 se debe registrar en las consultas periódicas, de manera más frecuente en niños con factores de riesgo 4,5,6.

Factores de riesgo identificados para la afección pediátrica son HAS paterna, ambos padres hipertensos, el 50\% de los hijos heredará la patología, ${ }^{2,3}$ si uno sólo lo es, baja al 33\%. El $40 \%$ de los pacientes hipertensos tienen hiperinsulinemia, se han encontrado niveles elevados de insulina en escolares y adolescentes obesos asociados a sensibilidad al sodio y aumento de la reactividad vascular, que los predisponen a la HAS. ${ }^{7}$
Efectos reversibles con la reducción del peso. La PA sistémica se correlaciona inversamente con el peso de nacimiento. La tendencia de los niños de bajo peso de nacimiento a hacer HAS estaría asociada a una resistencia a la insulina ${ }^{8}$, a hiperlipidemia y a la presencia de catecolaminas que estimularían la angiotensina II. ${ }^{9}$ Los individuos que nacen con bajo peso tienen ocho veces más alterado el test de tolerancia a la glucosa que los recién nacidos con peso normal.8 La alteración del metabolismo glucosa-insulina reduce la tasa de crecimiento fetal, lo que se traduce en bajo peso de nacimiento.

\section{MATERIALES Y METODOS}

El diseño fue un estudio retrospectivo descriptivo de prevalencias en donde se analizaron expedientes digitales de 5941 consultas en los servicios de cardiología y nefrología pediátrica del Hospital General de Zacatecas, de agosto 2013 a agosto 2015, en busca de pacientes pediátricos con HAS en el sistema computado del hospital denominado SIGHO, siglas determinadas por creadores del software.

El estudio realizado en el Hospital General de Zacatecas, perteneciente a los Servicios de Salud del estado de Zacatecas, uno de los tres sistemas de salud de México, aparte del medio privado. El cual es considerado el hospital de mayor capacidad y con mayor cantidad de servicios del estado con su categoría de segundo nivel, que además ofrece servicios de tercer nivel. Recibe una afluencia derivada de los 58 municipios que conforman la entidad federativa, la cual cuenta con una población de 1.579.209 hasta el año 2015. Además de atender personas procedentes de comunidades aledañas de otros estados como Jalisco, Durango y San Luis Potosí. Estimándose una atención a 3172 personas al día, en los distintos servicios pertenecientes al hospital, con 150 personas al día para el servicio más solicitado, urgencias, y 365 personas al día para consultas con especialistas y sub-especialistas.

Como población, se seleccionaron todos aquellos pacientes niños con diagnóstico de HAS, de las consultas de cardiología y nefrología pediátrica, y se les revisó género, edad, etiología, método de confirmación diagnóstica, así como el tratamiento indicado.

Para su desarrollo fue necesario del uso de computador central y administrativo del hospital y del software hospitalario SIGHO para identificar las consultas y los pacientes con los filtros específicos.

En lo que respecta a aspectos éticos, el actual trabajo fue aprobado por el comité de investigación de la institución, y de las autoridades administrativas del mismo. Se ha garantizado el anonimato de cada paciente, por medio del uso número de expedientes y folio de pa- 
ciente otorgado por la institución.

\section{Instrumentos}

Por medio de computadora personal (PC) a través del software SIGHO si identifico expedientes y pacientes, utilizando los filtros diagnósticos y por edad, así como especialidad para la identificación de los casos.

A través de una PC con cuenta habilitada para la búsqueda especializada de dispuso a analizar expedientes con un rango de 2 años, y filtros previamente descritos, capturando datos en otro dispositivo extrahospitalario con el software de análisis, culminando con la presentación de los datos por género, edad, etiología ,método de confirmación diagnóstica y tratamiento.

El análisis de datos se realizó a través del Software SPSS. $19^{\circledR}$ para Window.

\section{RESULTADOS}

La revisión retrospectiva del 26 de agosto de 2013 al 26 de agosto de 2015, nos arrojó como resultados 3277 consultas en Cardiopediatría, y 2664 en nefrología pediátrica con un total de 5941 consultas. Lo cual evidencia una mayor eficacia, en un principio, se diagnostica por parte del servicio de cardiología en contraposición con el servicio nefrológico. De las 5941 consultas corresponden sólo a 40 pacientes $(0,67 \%)$ con el diagnostico de HAS. Correspondiéndole al género femenino 15 individuos (37,5\%), y al masculino 25 (62,5\%).

De acuerdo a grupos de edad, véase Tabla 1. Datos que se lateralizan a menores edades y nos señalan una tendencia a alteraciones congénitas, que se denominan como la principal razón de HAS en niños, desplazando las HAS primaria, por la de causas secundarias, a diferencia de las estadísticas en adultos.

Respecto a la etiología secundaria de la HAS en nuestro estudio la Coartación de aorta se presentó como primera causa en 23 de los pacientes pediátricos con HAS; la insuficiencia renal crónica, y solo un niño con tumor renal (Ver Tabla 2).

Aquellos que se presentaron con diagnostico por coartación de aorta, 57,5\% de los pacientes; en todos ellos fue corroborado con ecocardiograma y angiotac. Y fueron intervenidos quirúrgicamente solo en 1 caso con stent y 8 de ellos con cirugía, 39,1\% de los casos con este diagnóstico, todos ellos presentes en arco ártico.

La terapéutica más utilizada en estos pacientes fue la farmacológica, siendo los fármacos más utilizados: Captopril; Espironolactona y Enalapril. Sin embargo no exenta el uso de otros medicamentos como lo fueron: Metoprolol, Nifedipino, Furosemida, Losartan (Ver Tabla 3).
De los 11 pacientes con insuficiencias renal crónica, fueron tratados la mayoría, $72,72 \%$ con un tratamiento alterno al ideal que es la Diálisis, y solo el 27,27\% con trasplante, que es considerado el tratamiento ideal para este tipo de pacientes, radicando su importancia en la corta edad del individuo. Importante mencionar que aquellos con coartación de aorta presentaron más comúnmente comorbilidades asociadas, principalmente al sistema cardiovascular, Hallándose 4 pacientes con estenosis valvular aortica, 17,3\%; 3 con persistencia del conducto arterioso al igual que el Asma, con 13,0\% para cada uno; 2 con rodete subaórtico, 8,69\%, y uno con insuficiencia mitral congénita, 4,34\%, ello de los pacientes con coartación. (Ver Tabla 4)

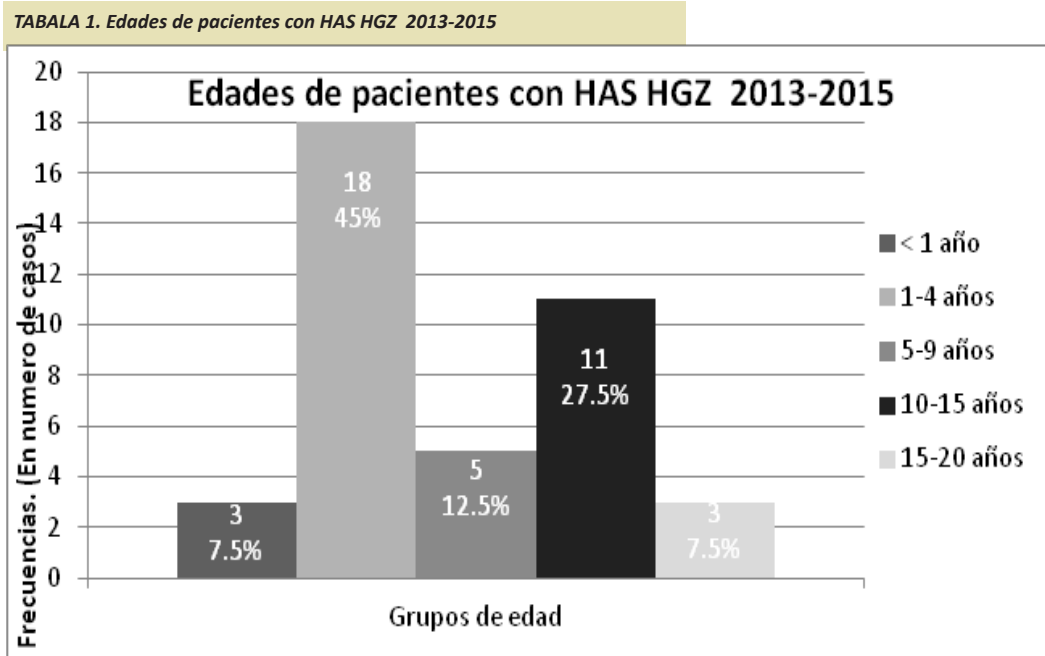

TABALA 2. Etiología HAS en pacientes HGZ 2013-2015

\section{Etiología HAS en pacientes HGZ}

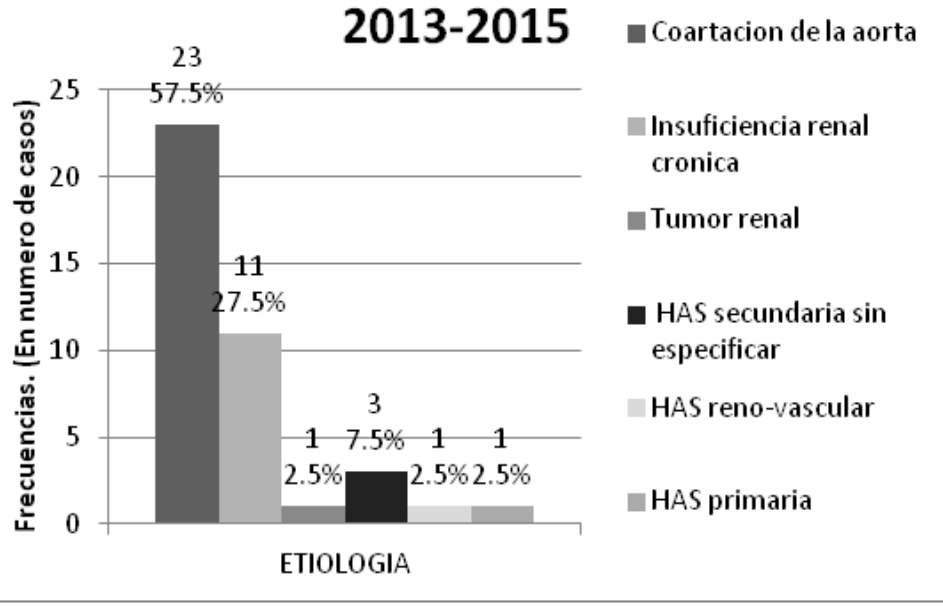


TABALA 3. Terapia farmacológica utilizada en HAS pediátrica HGZ 2013-2015.

Fármacos empleados en HAS pediátrica

17 HGZ 2013-2015.

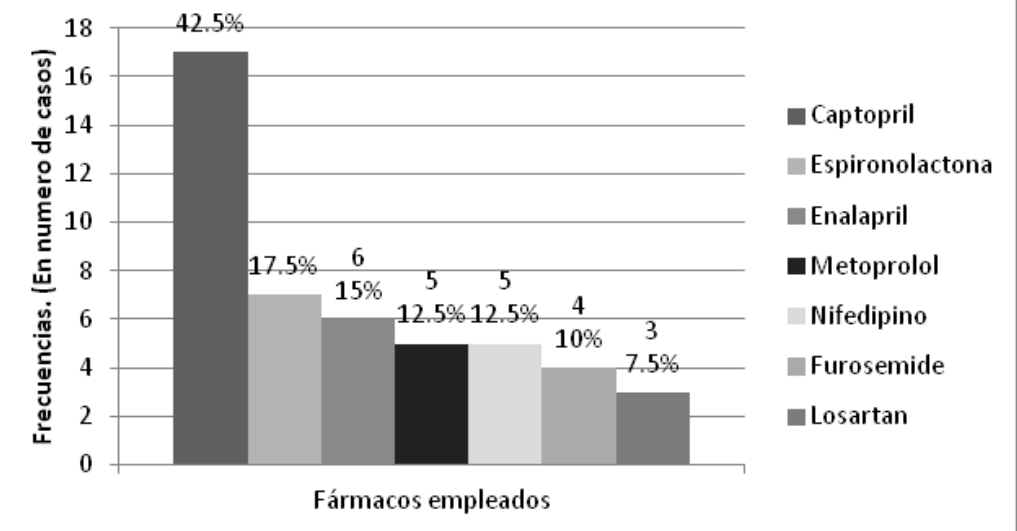

TABALA 4. Comorbilidades en coartación de aorta en pacientes con HAS en HGZ 2013-2015

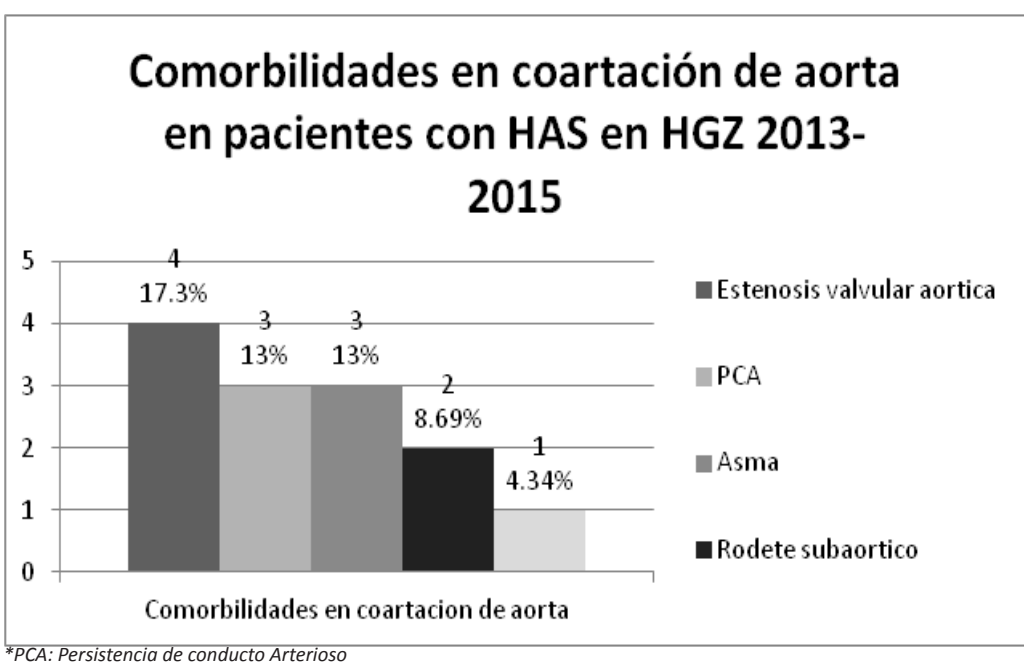

\section{DISCUSIÓN}

La literatura médica nos refiere las HAS como una enfermedad sub-diagnosticada en la actualidad, esto podría deberse en su mayoría a la falta de adiestramiento medico orientada a esta entidad1. Si bien su epidemiologia podría considerarse baja, en países en desarrollo como lo son los países de habla hispana en el nuevo mundo, se torna un punto importante a enfocar por parte de las autoridades de salud, poblaciones en las que la pirámide poblacional se encuentra ancha en las menores edades ${ }^{2,11,12,4,13,14}$. Lo anterior resalta la importancia por su efecto en la calidad de vida de los individuos que pronto se sumaran a la vida laboral activa y que arrastrando una calidad de enfermo crónico limitara su capacidad productiva, no solo impactando en el gasto de los servicios de salud ${ }^{3}$.
Lo primero de llamar la atención en este estudio, realizado en el Hospital General del Estado de Zacatecas, es la mínima prevalencia del padecimiento con un $0,67 \%$ de prácticamente 6 mil consultas en un hospital de alta concentración, de segundo nivel, comparativamente con la reportada por la literatura médica del $1,5 \%$ hasta un $3 \%{ }^{2,3}$ y que aun, se supone, sub-diagnosticada; además en los servicios a los que se canalizan esta patología. Lo que nos obliga a pensar en la cantidad de pacientes que pudiesen padecer este padecimiento y que aún no son diagnosticados y referidos a una institución de segundo y tercer nivel, donde se les pueda dar el tratamiento oportuno, debido a que en esta patología se suponen etiologías de gran repercusión en la calidad de vida del paciente, además de ser corregibles en el diagnóstico temprano, tales como coartación de la aorta, insuficiencia renal crónica y tumor renal, por mencionar algunos.

Sorpresivamente la etiología de los casos, en este estudio encontrados, invierten las causas reportadas en la literatura, encontramos en primer lugar de frecuencia la coartación de aorta y después otros problemas parenquimatosos renales y estenosis de la arteria renal, la cual es considerada la principal causa de HAS en niños por varios autores ${ }^{15,11}$. Ello nos obliga a pensar en la coartación de aorta como una causa muy frecuente de HAS en pacientes pediátricos específicamente en la zona del estado de Zacatecas y estados aledaños que puedan ser referidos a este hospital. Lo que justificaría en su defecto, la mayor cantidad diagnostica de esta entidad por parte del servicio de cardiología en nuestro medio.

Se reporta a la coartación aortica como causante del $5 \%$ de la HAS en niños, mientras en nuestra casuística de 40 pacientes, 23 (57,5\%) cursaban con ella, diagnosticados por ecocardiograma y Angiotac (Angiotomografia axial computalizada), métodos protocolarios establecidos por guías medicas españolas y de América latina,16,17,18. Resuelto mediante colocación de endoprotesis endovascular en un caso y 8 con cirugía, mientras que 14 continuaban con tratamiento farmacológico, observando cómo se encuentra desplazado el tratamiento primario por el farmacológico, esto ha de suponerse a los recursos propios hospitalarios, ya que por ser un hospital de servicios públicos la cantidad de recursos se ven limitados por la gran afluencia poblacional. ${ }^{18}$

La insuficiencia renal crónica, como etiología, estuvo presente en 11 pacientes que corresponde solo al $27,5 \%$, una cifra nada despreciable, sin embargo sobre de la causa principal de carácter renal referida en la literatura que es la estenosis de la arteria renal. De estos pacientes solo a 3 se les dio el beneficio del tratamiento ideal que es el trasplante, por razones las cuales no co- 
rresponden al objetivo de este trabajo.

Otro aspecto de importancia es el abordaje terapéutico, donde el grupo farmacéutico que más se usó fueron los inhibidores de la enzima convertidora de angiotensina (IECA), respaldado por recomendaciones recientes ${ }^{18}$, y aunque los, antagonistas de receptores de angiotensina (ARA) demuestran buenos resultados en otras revisiones 18,19 , no fueron parte de los fármacos de primera línea en el tratamiento de hipertensión arterial sistémica en pacientes pediátricos.

A pesar de que guías latinoamericanas refieren que el tratamiento farmacológico no debe ser la primera línea de tratamiento en este padecimiento ${ }^{18}$, es importante mencionar el cómo la etiología cambia por completo el enfoque del paciente, situación que se demuestra en este estudio. Debido a que sólo 1 paciente presentaba hipertensión arterial primaria, es el único candidato a un tratamiento no farmacológico. El tratamiento farmacológico está indicado en todos los pacientes que presenten HAS sintomática y secundaria ${ }^{20}$, lo que fundamenta el abordaje terapéutico.

Esto nos demuestra la importancia de la hipertensión arterial en el paciente pediátrico, debido a que será un dato realmente importante para el diagnóstico y sospecha alguna enfermedad asociada, tal como nos demuestra este estudio y la amplia literatura al tema. Para el grupo de estudio es de importancia resaltar la causa no renal de HAS en pacientes pediátricos y la alza causada por anormalidades cardiológicas, que es de importancia epidemiológica para la zona de estudio. Así como las medidas terapéuticas tomadas con las ideales según la literatura, desconociéndose las razones, tema ajeno en el cual no corresponde a los objetivos del estudio.

\section{REFERENCIAS}

1. González Sánchez Raquel, Llapur Milián René, Jiménez Hernández Juana María, Sánchez Pompa Addys. Percepción de los médicos de atención primaria de salud sobre el riesgo de hipertensión arterial en la infancia. Rev Cubana Pediatr de hipertensión arterial en la infancia. Rev Cubana Pediatr [Internet]. 2012 Jun [citado 2016 Jul 13] ; 84( 2): 155-164.
Acceso el 15 de octubre de 2016. Disponible en: http://scielo.sld. cu/pdf/ped/v84n2/ped05212.pdf

2. González-Reyes O, Alonso-Cordero M, Martín-Estévez L. Morbilidad por hipertensión arterial en niños y adolescentes. Revista de Ciencias Médicas de La Habana [revista en Internet]. 2011; de Ciencias Médicas de La Habana [revista en Internet]. 2011;
17(2). Acceso el 15 de octubre de 2016. Disponible en: http:// revcmhabana.sld.cu/index.php/rcmh/article/view/523/902

3. Canciano-Chirino E, Cruz-Suárez D, Sánchez-González P. Prehipertensión arterial en adolescentes, epidemiología y otras variables de riesgo para la adultez temprana. Revista de Ciencias Médicas de La Habana [revista en Internet]. 2011; 17(1). Acceso el 15 de octubre de 2016. Disponible en : http://revcmhabana.sld.cu/index.php/rcmh/article/view/499/861

4. Graciela Szera, Irina Kovalskysa, María J. De Gregorio. Prevalencia de sobrepeso, obesidad y su relación con hipertensión arterial y centralización del tejido adiposo en escolares. Arch. argent. pediatr. Buenos Aires, nov.-dic. 2010; vol.108 no.6. Acceso el 15 de octubre de 2016. Disponible en: http://www.sap.org.ar/ docs/publicaciones/archivosarg/2010/v108n6a04.pdf

5. M. Balas-Nakash, A. Villanueva-Quintana, S. Tawil-Dayan. Estudio piloto para la identificación de indicadores antropométricos asociados a marcadores de riesgo de síndrome metabólico en escolares mexicanos. Bol Med Hosp Infant Mex. 2008. Vol. 65, marzo-abril. Acceso el 15 de octubre de 2016. Disponible en: http://www.scielo.org.mx/pdf/bmim/v65n2/v65n2a4.pdf

6. Diaz BLA y cols. Circunstancias especiales: hipertensión arterial en niños, hipertensión arterial en el adulto mayor, hipertensión en el embarazo. Rev Mex Cardiol 2005; 16 (1): 35 41. Acceso el 15 de octubre de 2016. Disponible en: $h t t p: / / w w w$. medigraphic.com/pdfs/cardio/h-2005/h05If.pdf

7. Salcedo-Rocha Ana L, García de Alba Javier E, ContrerasMarmolejo Margarita. Presión arterial en adolescentes mexicanos: clasificación, factores de riesgo e importancia. Rev. salud nos: clasificacion, factores de riesgo e importancia. Rev. salud
pública. $2010 \mathrm{Aug} ; 12(4): 612-622$. Acceso el 15 de octubre pública. 2010 Aug ; 12( 4 ): 612-622. Acceso el 15 de octubre
de 2016. Disponible en: http://www.scielo.org.co/pdf/rsap/v12n4/ $v 12 n 4 a 08 . p d f$

8. Álvarez Ponce Vivian Asunción, Alonso Uría Rosa María Ballesté López Irka, Muñiz Rizo Milagros. El bajo peso al nace y su relación con la hipertensión arterial en el embarazo. Rev Cubana Obstet Ginecol. 2011 Mar; 37( 1 ): 23-31. Acceso el 15 de octubre de 2016. Disponible en: http://scielo.sld.cu/pdf/gin/ v37n1/gin05111.pdf

9. A. Díaz, M. Tringle, Et. Al. Control de la presión arterial y prevalencia de hipertensión arterial en niños y adolescentes de una población rural de Argentina. Datos preliminares del Proyecto Vela. Arch Argent Pediatr 2010;108(1):68-74. Acceso el 15 de octubre de 2016. Disponible en: http://s3.amazonaws.com/ academia.edu.documents/38200208/v108n1a12.pdf?AWSAccessK eyId=AKIAJ56TOJRTWSMTNPEA\&Expires $=1476585019 \&$ Sign eyId $=$ AKIAJ56TQJRTWSMTNPEA\&Expires $=1476585019 \&$ Sign
ature $=J$ Jic $6 Z \% 2$ Ft 4 XGllyKxf6V3FOvRaT0 $\% 3 D \&$ response-cont

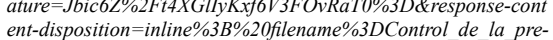
sion_arterial_y_prevale.pdf

10. Lurbe E., Cifkova R., Cruickshank J.K., Dillon M.J., Ferreira I., Invitti C., et al. Manejo de la hipertensión arterial en niños y adolescentes: recomendaciones de la Sociedad Europea de Hipertensión. An Pediatr (Barc). 2010;73(1):51.e1-51.e28 Acceso el 15 de octubre de 2016. Disponible en: http://www.sciencedirect. com/science/article/pii/S1695403310001694

11. Rodríguez-Herrera R, Carbajal-Rodríguez L, García-de la Puente S, Zarco-Román J, Perea-Martínez A. Hipertensión arterial sistémica en niños. Acta Pediatr Mex 2008;29(2):89-101. Acceso el 15 de octubre de 2016. Disponible en: http://www.medigraphic. com/pdfs/actpedmex/apm-2008/apm082g.pdf

12. Lagomarsino f edda, saieh a carlos, aglony i marlene. Recomendación de Ramas: Actualizaciones en el diagnóstico y tratamiento de la Hipertensión Arterial en Pediatría. Rama de Nefrología, Sociedad Chilena de Pediatría. Rev. chil. pediatr. 2008 Feb; 79(1): 63-81. Acceso el 15 de octubre de 2016. Disponible en: http://www.scielo.cl/pdf/rcp/v79n1/art10.pdf

13. Enrique Oliver Aregullin-Eligio, Ped; María Cándida AlcortaGarza, Ped. Prevalencia y factores de riesgo de hipertensión arterial en escolares mexicanos: caso Sabinas Hidalgo. Salud pública Méx. Cuernavaca ene.-feb. 2009; vol.51 no.1 Acceso el 15 de octubre de 2016. Disponible en: http://www.scielo.org.mx/pdf/ spm/v5ln1/05.pdf

14. National High Blood Pressure Education Program Working Group on High Blood Pressure in Children and Adolescents: Group on High Blood Pressure in Children and Adolescents: the fourth report on the diagnosis, evaluation, and treatment
of high blood pressure in children and adolescents. Pediatrics of high blood pressure in children and adolescents. Pediatrics 2004; 114: 555-576. Acceso del 15 de octubre del 2016. Dispo-
nible en: http://pediatrics.aappublications.org/content/pediatrics/114/Supplement_2/iv.full.pdf

15. Lomelí, C, Rosas M, Mendoza-Gonzalez C, Méndez A, Lorenzo $\mathrm{J}$, Buendía A et al. Hipertensión Arterial sistémica en el niño y adolescente. Archivos de Cardiología de México. Vol. 78 Supl. 2 Abril-Junio 2008:S2, 82-93 Acceso el 15 de octubre de 2016. Disponible en: http://www.scielo.org. $m x / p d f / a c m / v 78 s 2 / v 78 s 2 a 4$.pdf

16. Erbel R, Aboyans V, Boileau C, Bossene E, Di Bartolomeo R, Eggerbrecht H, et al. Guía ESC 2014 sobre diagnóstico y tratamiento de la patología de la aorta. Rev Esp Cardiol. 2015;68(3):242.e1-e69. Disponible en: http.//www.revespcardiol. 
org/es/comentarios-guia-practica-clinica-esc/artuculo/90387049

17. S. García de la Puente; S. Zaltzman G. Hipertensión arterial en niños. Acta pediátr. Méx. sept.-oct. 1994;15(5):233-48.

18. Simonetti G, Rizzi M, Donadini R, Bianchetti MG. Effects of antihypertensive drugs on blood pressure and proteinuria in childhood. J Hypertens. 2007;25:2370-6. Acceso el 15 de octubre del 2016. Disponible en: http://journals.lww.com/jhypertension/ Abstract/2007/12000/Effects of antihypertensive drugs on blood.2.aspx
19. Sánchez Ramiro A, Ayala Miryam, Baglivo Hugo, Velázquez Carlos, Burlando Guillermo, Kohlmann Oswaldo et al . Guías Latinoamericanas de Hipertensión Arterial. Rev Chil Cardiol. 2010; 29( 1 ): 117-144. Acceso el 15 de octubre de 2016. Disponible en: http://www.scielo.cl/pdf/rchcardiol/v29n1/art12.pdf

20

Medeiros Domingo Mara, Muñoz Arizpe Ricardo. Enfermedad renal en niños. Un problema de salud pública. Bol. Med. Hosp. Infant. Mex. 2011 Ago; 68( 4 ): 259-261. Acceso el 15 de octubre de 2016. Disponible en: $h t t p: / / w w w . s c i e l o . o r g . m x / p d f / b m i m /$ 688n4/v68n4a2.pdf 